\title{
Efecto de diferentes densidades de siembra sobre las características agronómicas del sésamo (Sesamum indicum L.) en el distrito de Curuguaty
}

\author{
Effect of different sowing densities on the agronomic characteristics \\ of sesame (Sesamum indicum L.) in the district of Curuguaty \\ Milciades Melgarejo A. ${ }^{1 *}$, Alberto Galeano ${ }^{1}$, Diosnel Amarilla M. ${ }^{1}$, Ever Maidana ${ }^{1}$, \\ Miguel Bogado', Rafael Franco ${ }^{1}$, Marcos J. Mendoza², Patricia Colman ${ }^{1}$, \\ Oscar Silvero ${ }^{1}$, Wilfrido D. Lugo 3 y Modesto Da Silva Oviedo 3
}

\begin{abstract}
RESUMEN
Con el objetivo de evaluar los efectos de diferentes densidades poblacionales sobre las características agronómicas del cultivo de sésamo (Sesamum indicum L.), se realizó este estudio en el distrito de Curuguaty-Paraguay en el periodo 2017/18, utilizando la variedad Escoba blanca. El diseño experimental usado fue el de bloques completos al azar con 6 tratamientos: 60, 80, 100, 120, 140 y 160 plantas por $\mathrm{m}^{2}$ con 4 repeticiones. La altura media de plantas fue de $263,80 \mathrm{~cm}$ sin diferencias estadísticas significativas entre tratamientos. Con 100 plantas por $\mathrm{m}^{2}$ se obtuvieron 458 cápsulas y fue diferente al tratamiento con 140 plantas por $\mathrm{m}^{2}$. El mayor número de ramos y mejor rendimiento se logró con 100 plantas por $\mathrm{m}^{2}$. Con 140 plantas por $\mathrm{m}^{2}$ se observó que el peso de mil granos llegó a 3,06 g y fue diferente al tratamiento con 160 plantas por $\mathrm{m}^{2}$.
\end{abstract}

Palabras clave: ajonjolí, escoba blanca, rendimiento.

\begin{abstract}
This study was conducted in the district of Curuguaty-Paraguay in the period 2017/18 to evaluate the effects of different population densities on sesame cultivation's agronomic characteristics using the Escoba Blanca variety (Sesamum indicum $L$.). The experimental design used was randomized complete blocks with 6 treatments; 60, 80, 100, 120, 140 and 160 plants per $m^{2}$ with 4 repetitions. The average height of plants was $263.80 \mathrm{~cm}$ without significant statistical differences between treatments. With 100 plants per $m^{2}, 458$ capsules were obtained, and it was different from the treatment with 140 plants per $m^{2}$. The highest number of branches and best productivity was observed with 100 plants per $\mathrm{m}^{2}$. With 140 plants per $\mathrm{m}^{2}$ it was observed that the weight of a thousand grains reached 3,06 $\mathrm{g}$ and was different from the treatment with 160 plants per $\mathrm{m}^{2}$.

Keywords: ajonjoli, escoba blanca, productivity.
\end{abstract}

\section{Introducción}

El sésamo o ajonjolí (Sesamum indicum L.) pertenece a la familia de las Pedaliaceae y es una de las oleaginosas cultivadas más antiguas (Weiss, 2000) e importantes del mundo. La semilla es utilizada como alimento nutritivo para el hombre y se destaca en la elaboración de productos horneados y de confitería. Según Abou-Gharbia et al. (1997), está compuesta por alrededor de 55\% de lípidos y $20 \%$ de proteínas, y además contiene vitaminas y minerales. El aceite producido del primer prensado en frío se encuentra entre los aceites comestibles más caros del mundo debido a su bajo tenor en colesterol y alto tenor en ácidos grasos poliinsaturados: contiene cerca del $47 \%$ de ácido oleico y $39 \%$ de ácido linoleico. El aceite del segundo prensado en caliente tiene, después

\footnotetext{
1 Facultad de Ciencias Agropecuarias y Ambientales. Departamento de Fitotecnia. Universidad Nacional de Canindeyú. Katueté, Paraguay. E-mail:agronomia@fcaa.edu.py

2 Corteva Agriscience. Asunción, Paraguay.

3 Facultad de Ciencias Agrarias. Universidad Nacional de Concepción. Concepción, Paraguay.

* Autor por correspondencia: milciadesmelgarejo1@gmail.com
}

Fecha de Recepción: 29 de mayo, 2019.

Fecha de Aceptación: 13 de febrero, 2020. 
de la extracción, una calidad inferior a la del aceite prensado en frío. Este aceite se utiliza para la producción de jabones, pinturas, cosméticos, tintas, así como en la industria farmacéutica y fabricación de pesticidas (Oplinger et al., 1990; Ram et al., 1990; Simon et al., 1984). Además, la torta obtenida posterior a la extracción del aceite es usada para la elaboración de ración animal, como suplemento alimenticio para humanos y como fertilizante. Otra de sus ventajas es la alta posibilidad de su utilización como fuente de biodiesel. Banapurmath et al. (2008) y Saydut et al. (2008) comprobaron la operatividad y calidad de este combustible. Los mayores productores mundiales de sésamo son Myanmar, India, China y Sudán, donde el 70-75 \% de la producción es utilizada para la extracción de aceite.

En Paraguay, el sésamo es uno de los principales rubros de la agricultura familiar. En el año 2018 se sembraron aproximadamente 48.000 hectáreas y los principales destinos de exportación fueron Japón, Europa, Oriente Medio y Estados Unidos.

El espaciado demasiado ancho conduce a una baja densidad de plantas por unidad de área y reduce la cobertura del suelo, mientras que un espacio demasiado estrecho está relacionado con la intensa competencia entre plantas por factores de crecimiento (Singh et al., 2004). además de influenciar en los costes. Rahnama y Bakhshandeh (2006) observaron que la variación en la densidad de siembra influyó en la cantidad de cápsulas por planta, peso de mil granos, rendimiento del cultivo. En otro estudio,
Ngala et al. (2013) comprobaron que diferentes densidades afectaron la altura de plantas, número de ramas por planta y rendimiento. Enciso et al. (2015) estudiaron el efecto de cuatro densidades poblacionales de siembra sobre el rendimiento de sésamo y observaron una disminución en el rendimiento con densidades poblacionales iguales o mayores a 125.000 plantas por hectárea.

El objetivo de este estudio fue evaluar el efecto de diferentes densidades poblacionales sobre las características agronómicas del cultivo de sésamo.

\section{Materiales y métodos}

El experimento de campo se realizó durante los años agrícolas 2017/18 en el predio de la Universidad Nacional de Canindeyú, localizado en el distrito de Curuguaty, Paraguay, según las siguiente coordenadas: $24^{\circ} 28^{\prime} 18^{\prime \prime}$ de latitud y $55^{\circ} 41^{\prime} 32$ " de longitud. El área donde se hizo el experimento posee un suelo clasificado como Rhodic Paleudult del orden Ultisol (López et al., 1995). El clima de la región es subtropical húmedo con temperatura media anual de $21,3^{\circ} \mathrm{C}$ y un promedio de $1.385 \mathrm{~mm}$ de lluvia anual (Fecoprod, 2016). El comportamiento de las condiciones climatológicas durante el desarrollo del cultivo se muestra en la Figura 1. El resultado del análisis químico del suelo del área en profundidad de $0-20 \mathrm{~cm}$, realizado antes de la instalación del experimento, presentó valores de $\mathrm{P}=8,40\left(\mathrm{mg} \mathrm{dm}^{-3}\right)$; M.O. $=10\left(\mathrm{~g} \mathrm{dm}^{-3}\right)$; $\mathrm{pH}\left(\mathrm{CaCl}_{2}\right)=6 ; \mathrm{H}+\mathrm{Al}=4,35\left(\mathrm{cmolc} \mathrm{dm}^{-3}\right) ; \mathrm{Al}^{3+}$

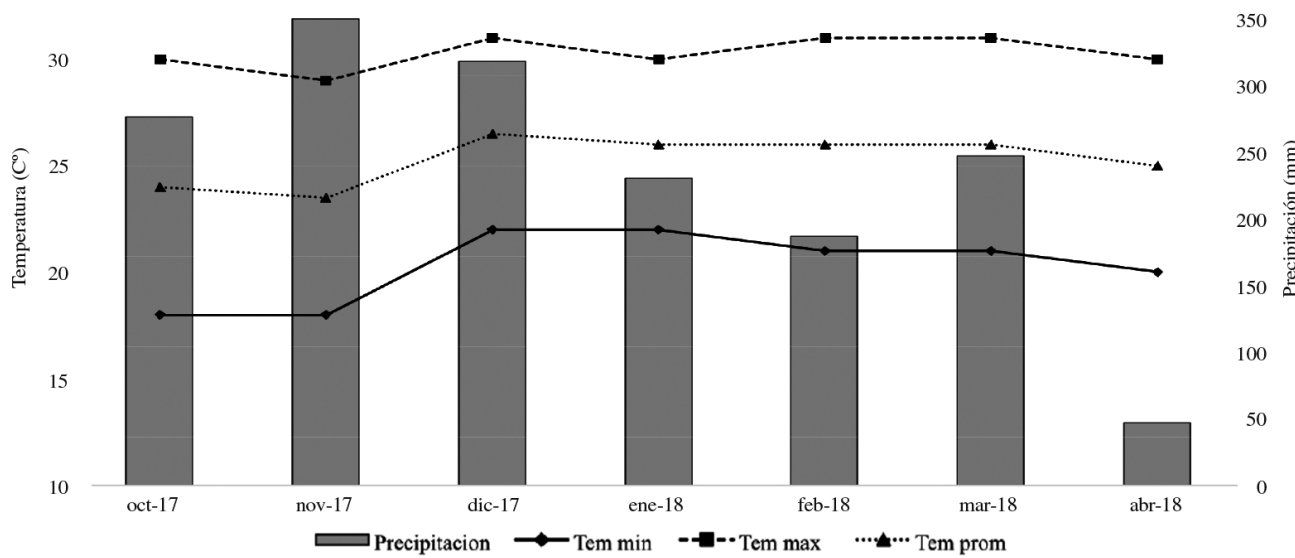

Figura 1. Precipitación, Temperatura mínima, media y máxima en el área del experimento en el periodo de octubre de 2017 a abril de 2018. Curuguaty. Paraguay. 
$=0,31\left(\mathrm{cmol}_{\mathrm{c}} \mathrm{dm}^{-3}\right) ; \mathrm{K}^{+}=0,13\left(\mathrm{cmol}_{\mathrm{c}} \mathrm{dm}^{-3}\right) ; \mathrm{Ca}^{+2}$ $=1,11\left(\mathrm{cmol}_{\mathrm{c}} \mathrm{dm}^{-3}\right) ; \mathrm{Mg}^{+2}=0,38\left(\mathrm{cmol}_{\mathrm{c}} \mathrm{dm}^{-3}\right)$.

El diseño experimental utilizado fue el de bloques completos al azar con seis tratamientos y cuatro repeticiones. Los tratamientos consistieron en $60,80,100,120,140$ y 160 plantas por metro cuadrado. La preparación de las parcelas se realizó un mes antes de la siembra con una rastreada. Una semana antes de la siembra se aplicó herbicida glifosato para dejar la parcela libre de malezas y proceder a la siembra. El experimento ocupó un área experimental total de $805 \mathrm{~m}^{2}$. La parcela o unidad experimental tenía $5 \mathrm{~m}$ de ancho y $5 \mathrm{~m}$ de largo, y contenía 5 líneas del cultivo con espaciamiento de $1 \mathrm{~m}$ entre sí. El área útil era de $12 \mathrm{~m}^{2}$ utilizando tres líneas centrales y eliminando una hilera de cada lado y $50 \mathrm{~cm}$ de los extremos por el efecto borde. La distancia entre bloques fue de 1 metro.

La variedad utilizada fue la Escoba blanca proporcionada por la empresa Shirosawa Company S.A.I.C. y presenta las siguientes características: ciclo vegetativo de 120 días, altura de 1,5 a $2 \mathrm{~m}$ de altura, con 1 a 20 ramas por planta, el color de la flor es blanco, ausencia de pubescencia, color de la cápsula en la etapa madura amarillo, cada planta puede tener de 100 a 800 unidades de cápsula, el color de la semilla es blanco o crema y el rendimiento varía entre 650 y $1.500 \mathrm{~kg} \mathrm{ha}^{-1}$ (Carreño, 2013).

Se utilizaron $200 \mathrm{~kg} \mathrm{ha}^{-1}$ de la formulación 15-15-15 para la fertilización del suelo, según resultados del análisis químico y recomendación del cultivo. El manejo de plantas dañinas se hizo por medio de carpidas manuales realizadas conforme a la incidencia durante el experimento. No se observaron enfermedades o insectos en el estudio. El experimento fue ejecutado sin sistema de irrigación. La siembra se realizó en fecha 10/10/17 de forma manual abriendo cinco surcos distanciados a 1 metro. La profundidad de siembra fue de $3 \mathrm{~cm}$. Un mes después de la emergencia se hizo el raleo de plántulas para el ajuste de las densidades correspondientes por tratamiento.

La altura de plantas fue evaluada en el momento de la cosecha, donde se midió desde la superficie del suelo hasta el ápice de la planta utilizando una cinta métrica graduada y calculando el promedio de diez plantas del área útil. Para evaluar el número de cápsulas y el número de ramas por planta se seleccionaron de forma aleatoria diez plantas del área útil y se contaron todas las cápsulas y ramas para luego promediarlos.
La cosecha manual se realizó cuando las plantas de sésamo alcanzaron su estadio de maduración fisiológico y apertura de las primeras cápsulas inferiores, 110 días posteriores a la siembra y considerando todas las plantas del área útil de cada parcela. Las plantas fueron cortadas a una altura de $15 \mathrm{~cm}$ del suelo utilizando machete. Luego se formaron mazos de 15 a $20 \mathrm{~cm}$ de diámetro con las plantas cortadas. Seguidamente se formaron parvas utilizando 4 a 6 mazos de tal manera que las cápsulas quedaran hacia arriba para exponerlos al sol durante 15 días. La trilla se realizó una vez observado que las cápsulas estaban abiertas disponiéndolos encima de una carpa. Para la limpieza de los granos se utilizaron tamices. $\mathrm{La}$ determinación de la masa de 1.000 granos se hizo conforme a la metodología descrita en las Regras de Análise de Sementes (BRASIL, 2009), donde ocho muestras con 100 granos de cada repetición se pesaron con balanza analítica de precisión y luego se calculó el promedio de la masa de mil granos. Los granos del área útil de cada unidad experimental se pesaron con balanza de precisión de cuatro decimales y el rendimiento se expresó en $\mathrm{kg} \mathrm{ha}^{-1}$.

Las variables altura de plantas, número de cápsulas por planta y peso de mil granos fueron sometidas al análisis de varianza, donde se aplicó el test $\mathrm{F}$ al nivel de 0.05 de probabilidad y test de Tukey al 0.05 de probabilidad para las comparaciones de medias. Las variables número de ramas por planta y rendimiento fueron sometidas al análisis de regresión.

\section{Resultados y discusión}

Con relación a la altura de plantas (ADP) de sésamo, no se observaron diferencias estadísticas significativas $(p>0,05)$ y se obtuvo una media de $263.83 \mathrm{~cm}$ (Tabla 1). Este resultado coincide con Zarate et al. (2011) con media de $229 \mathrm{~cm}$ sin diferencias estadísticas entre densidades. Beltrâo et al. (2001) mencionan que las variedades clasificadas como gigante y crecimiento indeterminado pueden llegar hasta $300 \mathrm{~cm}$ de altura, y es el grupo al cual pertenece la Escoba blanca.

El número de ramas por planta fue afectado significativamente por las diferentes densidades de siembra de sésamo, las cuales se ajustaron a la expresión polinomial de segundo grado, donde 
el mejor resultado estimativo se observaría con 83 por $\mathrm{m}^{2}$. Densidades mayores arrojaron como resultado una disminución de ramas (Figura 2). Van Humbeeck y Oviedo (2012) realizaron estudios con sésamo utilizando 6 densidades de siembra, y el tratamiento con mayor número de ramas fue el de 50 plantas por $\mathrm{m}^{2}$ con 7,45 ramas. Las plantas que poseen un elevado número de ramas reflejan un aumento del número de cápsulas.

Se observaron diferencias estadísticas significativas $(p<0,05)$ para la variable número de cápsulas por planta (NDC) sembradas en diferentes densidades. Con 100 plantas por $\mathrm{m}^{2}$ se produjeron 458 cápsulas y la menor cantidad se obtuvo con la densidad de 140 plantas por $\mathrm{m}^{2}$ lográndose 346 unidades (Tabla 1). Al respecto, Mazzani (1999) indica que el número de cápsulas por planta es uno de los componentes que más afectan el rendimiento final. Sin embargo, no se observaron diferencias entre las densidades 60, 80,120 y 160 plantas por $\mathrm{m}^{2}$. Van Humbeeck y Oviedo (2012) consiguieron los mejores resultados sembrando 50 plantas por $\mathrm{m}^{2}$ donde obtuvieron 220 cápsulas por planta utilizando seis densidades. En el periodo de ejecución del experimento se observó un volumen elevado y buena distribución de lluvias (Gráfico 1), y fueron suficientes para que la humedad del suelo atendiera satisfactoriamente la exigencia hídrica del sésamo, lo que podría explicar la alta cantidad de cápsulas.

Se hallaron diferencias estadísticas significativas para la variable peso de mil granos (PMG). Con la densidad 140 plantas por $\mathrm{m}^{2}$ el peso de los granos llegó a 3,06 g, y únicamente fue diferente al tratamiento con 160 plantas por $\mathrm{m}^{2}$. No se observaron diferencias entre los tratamientos 60, 80, 100 y 120 plantas por $\mathrm{m}^{2}$. (Tabla 1). Van Humbeeck y Oviedo (2012) reportaron una media de 3,21 g sin encontrar diferencias estadísticas entre distintas densidades. El peso de mil granos puede variar en un rango de 2,9 a 3,3 g. Oviedo de Cristaldo (2008) observó una media de $3,2 \mathrm{~g}$.

El rendimiento del sésamo fue afectado significativamente por las densidades de siembra y la respuesta se ajustó a la regresión polinomial de segundo grado. Para esta variable, el máximo rendimiento se reportó en la densidad estimada de 117 plantas por $\mathrm{m}^{2}$ con posterior reducción (Figura 3). Asimismo, Enciso et al. (2015) estudiaron cuatro densidades de siembra de sésamo y registraron un descenso del rendimiento con densidades poblacionales iguales o mayores

Tabla 1. Altura de plantas (ADP), número de cápsulas (NDC) y peso de mil granos (PMG) de sésamo sembrado en el distrito de Curuguaty en la campaña 2017/18.

\begin{tabular}{clll}
\hline Plantas por $\mathrm{m}^{2}$ & ADP $(\mathrm{cm})$ & NDC & PMG $(\mathrm{g})$ \\
\hline 60 & 263 & $427 \mathrm{ab}$ & $2,78 \mathrm{ab}$ \\
80 & 263 & $410 \mathrm{ab}$ & $3,00 \mathrm{ab}$ \\
100 & 269 & $458 \mathrm{a}$ & $2,79 \mathrm{ab}$ \\
120 & 257 & $372 \mathrm{ab}$ & $2,76 \mathrm{ab}$ \\
140 & 264 & $346 \mathrm{~b}$ & $3,06 \mathrm{a}$ \\
160 & 267 & $439 \mathrm{ab}$ & $2,74 \mathrm{~b}$ \\
Media & 263,83 & 408,2 & 2,85 \\
Valor de F & $0,34^{\mathrm{ns}}$ & 3,31 & 4,39 \\
CV $(\%)$ & 5,31 & 11,43 & 4,56 \\
\hline
\end{tabular}

Medias en las columnas seguidas por letras iguales no difieren entre sí al nivel de significancia de 0,05 por el test de Tukey. Ns: no significativo.

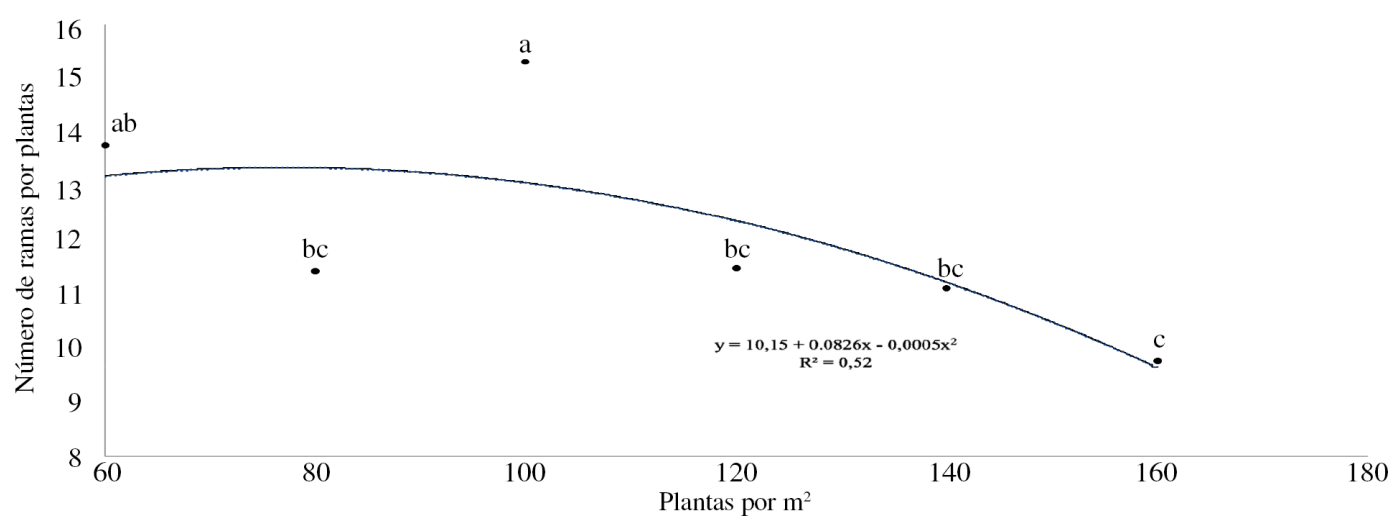

Figura 2. Número de ramas de sésamo en función de diferentes densidades de siembra de sésamo en la campaña 2017/18. Curuguaty. Paraguay. 


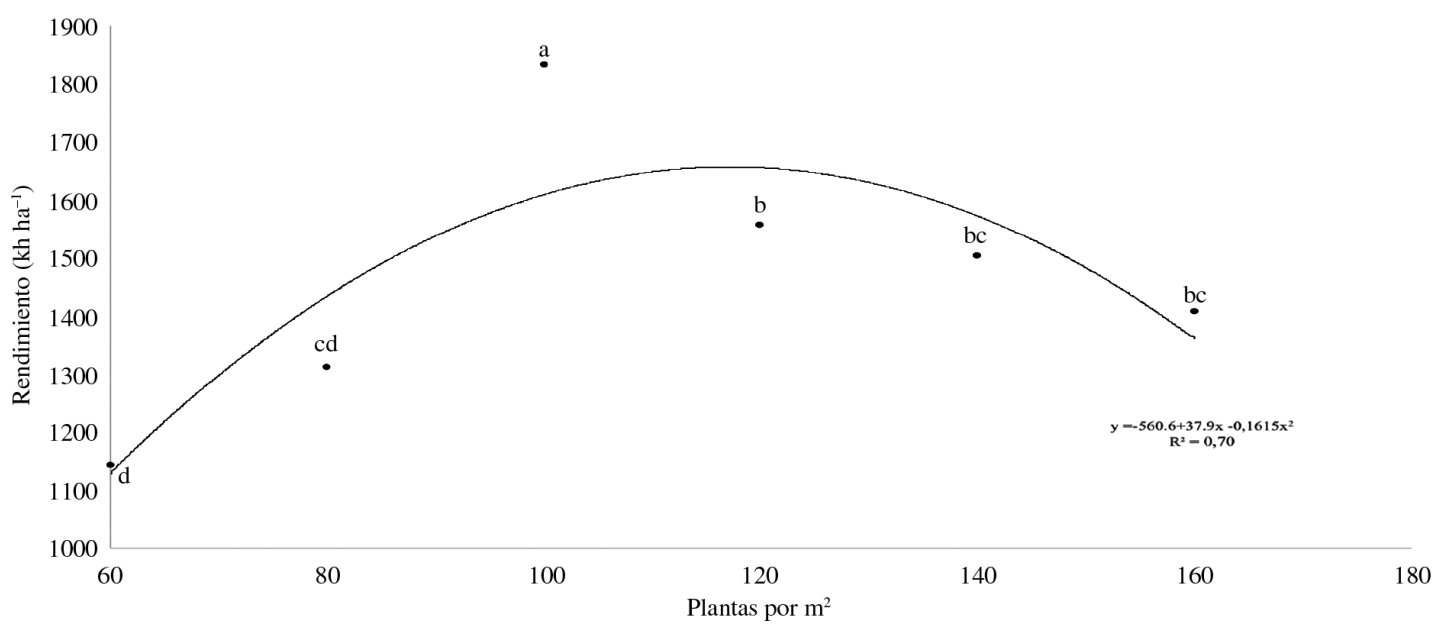

Figura 3. Rendimiento del sésamo en función de diferentes densidades de siembra de sésamo en la campaña 2017/18. Curuguaty. Paraguay.

a 125 plantas por $\mathrm{m}^{2}$. Por otro lado, Carreño y Rojas (2013) y Zárate et al. (2011) observaron una disminución del rendimiento del cultivo del sésamo a partir de 160 y 100 plantas por $\mathrm{m}^{2}$. El rendimiento del sésamo es una de las características que más varían. DISE (2003) y Cristaldo (2007) encontraron variaciones dentro del rango de 900 a $1.500 \mathrm{~kg} \mathrm{ha}^{-1}$.

\section{Conclusiones}

Para las densidades estudiadas, el mejor rendimiento y el mayor número de ramos se obtuvo con 100 plantas por $\mathrm{m}^{2}$.
El mayor número de cápsulas se observó con 100 plantas por $\mathrm{m}^{2} \mathrm{y}$ fue diferente a la densidad con 140 plantas por $\mathrm{m}^{2}$.

En relación con el peso de mil granos, con la densidad de 140 plantas por $\mathrm{m}^{2}$ se obtuvo $3,06 \mathrm{~g}$ y fue diferente a la densidad de 160 plantas.

\section{Agradecimientos}

Los autores agradecen a la Universidad Nacional de Canindeyú, a la Facultad de Ciencias Agropecuarias y Ambientales y al Programa Nacional de Incentivo a los Investigadores (PRONII) del Consejo Nacional de Ciencias y Tecnología (CONACYT).

\section{Literatura Citada}

Abou-Gharbia, H.A., Shahidi, F., Shehata, A.A.Y.; Youssef, M.M. 1997. Effects of processing on oxidative stability of sesame oil extracted from intact and dehulled seeds. Journal of the American Oil Chemists Society, 74, № 3, 215.

Abou-Gharbia, H.A.; Shehata, A.A.Y.; Shahidi, F.

2000. Effect of processing on oxidative stability and lipid classes of sesame oil. Food Research International, 33, $\mathrm{N}^{\circ} 5,330$.

Banapurmath, N.R.; Tewari, P.G.; Hosmath R.S.

2008. Performance and emission characteristics of a DI compression ignition engine operated on Honge, Jatropha and sesame oil methyl esters. Renewable Energy 33: 1982-1988.

Beltrâo, N.E. DE M.; Vieria, D.J.

2001. O Agronegócio do gergelim no Brasil. Brasília, D.F.; Brasil; EMBRAPA. 348 p.

BRASIL, Ministério da Agricultura, Pecuária e Abastecimento. 2009. Regras para análise de sementes. Secretaria de Defesa Agropecuária. Brasília: Mapa/ACS. 399 p.
Carreño, B.; Rojas, R.

2013. Densidades de siembra de la variedad escoba blanca de sésamo (Sesamum indicum L.),evaluada bajo el sistema chaqueado en la comunidad de Limones, municipio de Robore del departamento de Santa Cruz. Cámara Boliviana de Exportadores de Sésamo, 1-15.

Cristaldo, $\mathrm{R}$.

2007. Introducción y selección de cultivares de sésamo. In: Jornada Técnica-Científica del Cultivo de Sésamo. San Lorenzo, Campus Universitario: p. 2.

DISE. Dirección de semillas. Resolución No 427/03.

2003. Resolución del Ministerio de Agricultura y Ganadería, por la cual se dispone la inscripción de oficio en el Registro Nacional de Cultivares Comerciales las variedades de Sésamo, Escoba Blanca, Dorado y Negro de la especie (Sesamum indicum L). Mamani, J.; Aguirre, R.

2013. Evaluación del efecto de diversas densidades de siembra en el cultivo de soya (Glycine max(1) merril), en 
la comunidad de Canandoa 1, Municipio de San Pedro, campaña de invierno 2013, Revista Universidad, Ciencia y Sociedad. pp. 24-33.

Mazzani, B.

1999. Investigación y tecnología del cultivo del ajonjolí en Venezuela (en línea). Maracay, VE. Consultado el 6 mayo 2020. Disponible en http://ajonjolí.Sian.info.ve/toc.html.

Ngala, A.L.; Duge, I.Y. and Yakubu, H.

2013. Effects of inter-row spacing and plant density on performance of sesame (Sesame indicum L.) in a Nigerian Sudan Savanna. Science of International, 25(3): 513-519.

Rahnama, A. and Bakhshandeh, A.

2006. Determination of Optimum Row Spacing and Plant Density for Uni-Branched Sesame in Khuzestan Province. Journal of Agricultural Science and Technology, 8, 25-33.

Ram, R.; D. Catlin, J; Romero and C. Cowley.

1990. Sesame: New approaches for crop improvement. Advances in New Crops: 225-228.
Saydut, A.; Duz, M.Z.; Kaya, C.; Kafadar, A.B.; Hamamci C. 2008. Transesterified sesame (Sesamum indicum L.) seed oil as a biodiesel fuel. Bioresource Technology 99: 6656-6660.

Simon, J.E.; Chadwick y Craker, L.E.

1984. Herbs: An Indexed Bibliography. 1971-1980. The Scientific Literature in Selected Herbs and Aromatic and Medicinal Plants of the Temperate Zone. Archon Books, 770 pp., Hamden, CT.

Van Humbeeck A. Marcos A., Oviedo de C, Rosa María. 2012. Población de plantas y su efecto en el desarrollo vegetativo y rendimiento del sésamo (Sesamum indicum L.) variedad Escoba. Investigación Agraria. Universidad Nacional de Asunción. Investigación Agraria. pp. 25-30.

Van Rheenen, H.A.

1973. Major problems of growing sesame (Sesamum indicum L.) in Nigeria. Wageningen. Netherland, 73(12): 130.

Weiss, E.A.

2000. Sesame, Oilseed Crops. Longman Inc., New York, p. 131-164. 